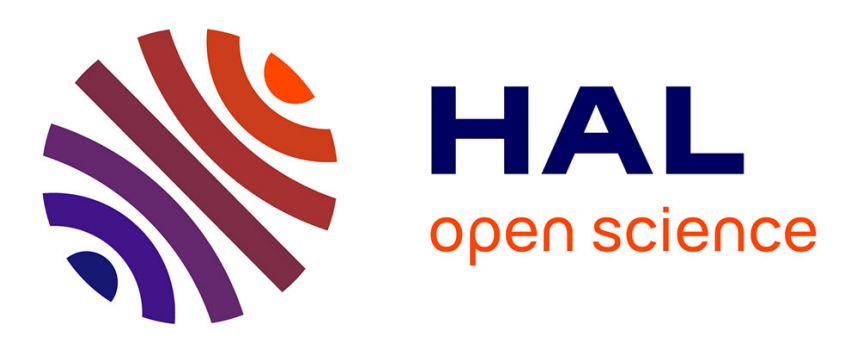

\title{
Single vs. two-photon microscopy for label free intrinsic tissue studies in the UV light region
}

\author{
Vitalijs Zubkovs, Frédéric Jamme, Slavka Kascakova, Franck Chiappini, \\ François Le Naour, Matthieu M. Refregiers
}

\section{- To cite this version:}

Vitalijs Zubkovs, Frédéric Jamme, Slavka Kascakova, Franck Chiappini, François Le Naour, et al.. Single vs. two-photon microscopy for label free intrinsic tissue studies in the UV light region. Analyst, 2014, 139, pp.2663-2667. 10.1039/c4an00203b . hal-01570708

\section{HAL Id: hal-01570708 \\ https://hal.science/hal-01570708}

Submitted on 1 Aug 2017

HAL is a multi-disciplinary open access archive for the deposit and dissemination of scientific research documents, whether they are published or not. The documents may come from teaching and research institutions in France or abroad, or from public or private research centers.
L'archive ouverte pluridisciplinaire HAL, est destinée au dépôt et à la diffusion de documents scientifiques de niveau recherche, publiés ou non, émanant des établissements d'enseignement et de recherche français ou étrangers, des laboratoires publics ou privés. 


\title{
Single vs. two photon microscopy for label free intrinsic tissue studies in UV light region
}

\author{
Vitalijs Zubkovs ${ }^{a, b}$, Frédéric Jamme ${ }^{a}$, Slavka Kascakova ${ }^{c, d}$, Franck Chiappini ${ }^{c, d}$, François Le Naour ${ }^{c, d}$ and \\ Matthieu Réfrégiers ${ }^{a, *}$
}

Fibrillar distribution in rat tail tendon and mice liver can be measured with optical methods. Two-photon excitation gives easy assessment of fibrotic collagen type I and II. Single ${ }_{10}$ photon deep ultraviolet (DUV) excitation imaging highlights all collagen types without discrimination. Their combination on the same tissue area provides a better overview of collagens in fibrillar diseases.

\section{${ }_{15}$ Introduction}

Two-photon microscopy is a fast developing tool for cell biology and tissue diagnosis. ${ }^{1-4}$ Its efficiency for imaging tissues in depth has been demonstrated and well recognized. ${ }^{4}$ Due to its intrinsic non-linear nature it shines two type of signals, i) second harmonic ${ }_{20}$ generated (SHG) diffusion that brings easy localization of the non-centrosymmetric structures present in biological materials with specific information concerning their orientation; ii) using near infrared excitation, it allows to image the ultraviolet excited autofluorescence of biological samples ${ }^{6}$ so called two-photon ${ }_{25}$ excitation fluorescence (TPEF). It is one of the possible methods to image label free biological samples with a great selectivity. SHG imaging can be applied for visualization of collagen ${ }^{7}$, which is a key component of load-bearing tissues (bones, tendons, etc.) and the most abundant protein in human body ( $30 \%$ of proteins 30 present in body mass ${ }^{1}$ ). Abnormalities in collagen development in several organs are often caused by a progression of inflammatory diseases and can be used as a pathological signature. SHG microscopy can be used to grade fibrosis in biological tissues. It should be highlighted that only fibrillar collagen types might be 35 detected by SHG microscopy. Therefore globular collagen types do not give any SHG signal. ${ }^{1,3}$

On the other hand, ultraviolet excitable autofluorescent compounds can be imaged using single photon ultraviolet (UV) light in cells and tissues ${ }^{8,9}$. UV fluorescence microscopy in deep 40 ultraviolet (DUV) range $(200-300 \mathrm{~nm})$ is an excellent tool for non-destructive qualitative specimen analysis.

Two-Photon Excitation Fluorescence (TPEF) microscopy can also be applied for autofluorescent imaging of endogenous compounds. But this technique cannot compete with 45 monophotonic microscopies in discreteness of a recorded spectrum, despite same range lateral resolution. ${ }^{10}$

We describe for the first time the complementarities and synergies between ultraviolet single photon and two-photon excitation of autofluorescent compounds in tissues and compare 50 the resolution and penetration depth of both modalities. Rat-tail as positive control and mice liver as unknown sample were studied and the potential of the combination was explored.

\section{Methods and Materials \\ DUV Synchrotron light imaging}

55 The full field synchrotron DUV imaging set-up is build around a Zeiss Axio Observer (Carl Zeiss, France) inverted microscope constructed with quartz-only optics. The white beam of DISCO beamline at Synchrotron SOLEIL ${ }^{11}$ is monochromatized by an iHR320 (Jobin-Yvon Horiba, Longjumeau, France) before 60 coupling with the entrance of a modified Zeiss Axio Observer Z1 (Carl Zeiss, Germany), monochromatic beam was chosen in order to determine composition of collagen in biological tissues exciting close to the $290 \mathrm{~nm}$ maximum, tryptophan and tyrosine were excited with the same excitation wavelength. A sharp ${ }_{65}$ dichroic mirror transmitting only above $300 \mathrm{~nm}$ (Omega Optical, Brattleboro, Vermont) reflected the incident light that was further focused onto the sample through a Zeiss Ultrafluar 100x (N.A. 1.25 , glycerine immersion). ${ }^{8}$ Emission was recorded with a Pixis 1024-BUV (Princeton Instruments, USA) camera after passing 70 through a series of bandpass filters (Semrock, Rochester, USA). Fluorescence images were typically recorded in few seconds exposure (Table 1; DUV1 and DUV2). For bead's stack imaging, $\mathrm{Z}$ scanning was performed with a PInano $\mathrm{Z}$ piezo slide scanner stage (PI, Germany). The whole system was controlled via ${ }_{75} \mu$ Manager. ${ }^{12}$

\section{Two-photon imaging microscopy}

The samples were analyzed with a Nikon A1MP+ confocal microscope (NIKON, France). A tuneable Maï Taï XF Ti:Saphire mode-locked laser (SPECTRA PHYSICS, France) excited the 80 sample between 710 and $920 \mathrm{~nm}$ through a 40x water immersion objective (N.A. 1.25, water immersion). Reflected second harmonic generated signal were detected at half the excitation wavelength with ad-hoc filters in front of the NDD GaAsP detectors. Excitation wavelength, acquisition time, and filters sets 85 were chosen in order to maximize resolution and signal intensity in each channel (Table 1; rows IR1 to IR2).

Table 1. Microscopes acquisition parameters.

\begin{tabular}{|c|c|c|c|c|c|}
\hline Number & $\begin{array}{c}\text { Excitation } \\
\text { wavelength } \\
(\mathrm{nm})\end{array}$ & $\begin{array}{l}\text { Emission } \\
\text { filter (nm) }\end{array}$ & $\begin{array}{c}\text { Image } \\
\text { acquisition } \\
\text { time (s) }\end{array}$ & $\begin{array}{l}\text { Targeted } \\
\text { compounds }\end{array}$ & $\begin{array}{l}\text { Co-excited } \\
\text { compounds }\end{array}$ \\
\hline DUV1 & 290 & $327-353$ & 5 & Tryptophan & $\begin{array}{c}\text { Tyrosine, } \\
\text { Pyridoxine, } \\
\text { Collagen }\end{array}$ \\
\hline DUV2 & 290 & $412-438$ & 5 & Collagen & $\begin{array}{c}\text { Elastin, } \\
\text { NADH, } \\
\text { Pyridoxine }\end{array}$ \\
\hline IR1 & 810 & $399-414$ & 32 & $\begin{array}{c}\text { Collagen } \\
\text { (SHG) }\end{array}$ & \\
\hline IR2 & 810 & $447-472$ & 32 & $\begin{array}{c}\text { NADH, } \\
\text { FAD (TPEF) }\end{array}$ & \\
\hline
\end{tabular}

Polystyrene beads 
Monodisperse $220 \mathrm{~nm}$ polystyrene beads from the polybeads sampler kit III (Polysciences, France) were deposited and dried on a quartz coverslip before observation. They give a very convenient size distribution standard $(220 \pm 20 \mathrm{~nm})$ for ultraviolet 5 fluorescence microscopy due to the natural autofluorescence of polystyrene around $390 \mathrm{~nm}$ under DUV excitation.

Rat tail tendon

Rat tendons have been studied with different methods (SHG microscopy, Scanning Electron Microscopy (SEM), 10 spectroscopy, chemical methods, etc.) and biochemical composition and their fibre organization is well characterized. ${ }^{13-15}$ This tissue is rich in collagen type I fibrils making it an excellent sample for comparison of microscopy techniques. Rat-tail tendons were taken from Sprague-Dawley rats following EU 151986 procedures. Tendons were deposited on slides after physiological serum rinsing.

Mice liver histological sections

The study has been focused on liver samples from a mouse model fed on chow diet. The mice were grown in the animal facility of

20 Institut André Lwoff (Villejuif, France). Mice were sacrificed after 5 weeks of breeding.

For SHG and DUV analysis fresh liver samples were fixed in formaldehyde and dehydrated in alcohol of increasing concentration up to absolute alcohol and finally immersed into 25 toluene. Thereafter, the tissue was embedded in liquid paraffin, solidified and sectioned at 10,20 $\mu \mathrm{m}$ with the microtome. Slices were mounted on quartz coverslips (thickness $0.17 \mathrm{~mm}$ ) by addition of a drop of distilled water. In the standard sample preparation protocol, the human serum albumin is usually used.

${ }_{30}$ However using the UV excitation the addition of human serum albumin can lead to parasite signal. Therefore we excluded the albumin from preparation protocol. The tissues were well preserved but presented several cracks formed during the segmentation procedure.

\section{${ }_{35}$ Results and Discussion}

\section{Comparing resolutions}

The usual way to compare the resolution of microscopes is to use calibrated nanobeads with fluorescent probes excited in the energy range of interest. In our case, for ultraviolet excitation, the

40 best compromise we found was to use the natural fluorescence of polystyrene beads of known size.
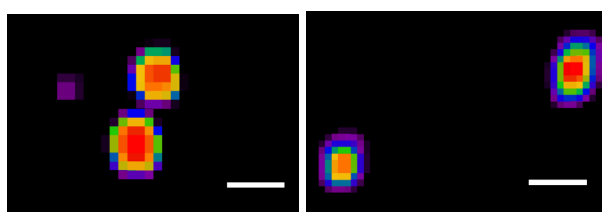

Figure 1. $220 \mathrm{~nm}$ polystyrene beads fluorescence imaging showing the resolving power of each microscopy technique. (left) $390 \mathrm{~nm}$

45 fluorescence after DUV excitation at $290 \mathrm{~nm}$ excitation. (right) TPEF image at $376 \mathrm{~nm}$ after IR $710 \mathrm{~nm}$ excitation. Scale bars $0.5 \mu \mathrm{m}$.

As seen on Fig. 1, the observed beads size after classic maximum likelihood estimation deconvolution is very close with both setups. Indeed, the natural confocal excitation provided by TPEF 50 is compensated by the very low $(290 \mathrm{~nm})$ excitation wavelength of widefield DUV fluorescence microscopy. Therefore, for all the following images, we can directly compare the information content of TPEF and DUV images.

\section{UV imaging of intrinsic tissues}

\section{${ }_{55}$ Rat-tail}

Before studying liver tissues samples, we wanted to compare the different modalities with a simple tissue, namely rat tail tendon which is known to be rich in type I collagen. ${ }^{15}$ The imaging area was chosen randomly during the first acquisition. It should be 60 mentioned that both imaging modalities are non-invasive for biological tissues and can therefore be applied in random order.

The abundance of types I, II collagen in rat tail tendon is
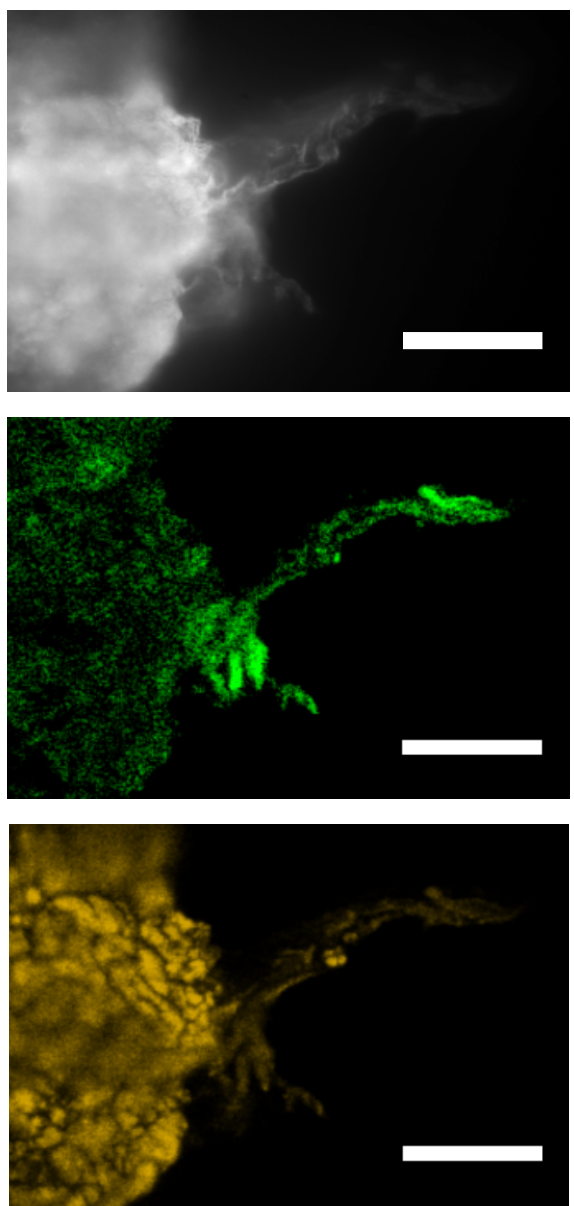

Figure 2. Rat-tail tendon multimodal imaging: Co-localized region of rattail tendon showing separate collagen fibres magnified. Collagen excitation at $290 \mathrm{~nm}$ in DUV, emission 412 - $438 \mathrm{~nm}$. (upper) and collagen fibres visualization using SHG microscopy. (middle) and TPEF intrinsic fluorescence $(447-472 \mathrm{~nm})$ excited at $810 \mathrm{~nm}$. (bottom) images. Scale bars $25 \mu \mathrm{m}$.

highlighted by SHG microscopy (figure 2, middle). In the tendon 70 collagen architecture, two types of features can be highlighted: elongated, wave-like fibres and curved interwoven fibrils. ${ }^{14}$ TPEF signal can be recorded simultaneously with SHG signal using higher wavelength bandpass filter sets, however, compared to single photon DUV excited fluorescence, TPEF excitation profile 75 is less structured and selective. ${ }^{16}$ It represents NADH and FAD distribution (table 1).

The same sample was transferred onto the DUV microscope for examination of collagen. On the figure 2, similarities in 
organization of collagen bundles can be noticed between DUV fluorescence and SHG images. Separate collagen fibres bundles are well distinguishable, however, in cellular regions DUV fluorescence shows several other collagen fibres types which results in higher concentration (around 10\% of all collagen types ${ }^{15}$ in rat tail tendon).

Mice liver microscopy

As shown before on rat-tail tendon, collagen localization can be highlighted with DUV microscopy (Fig. 2) but cannot be typed.
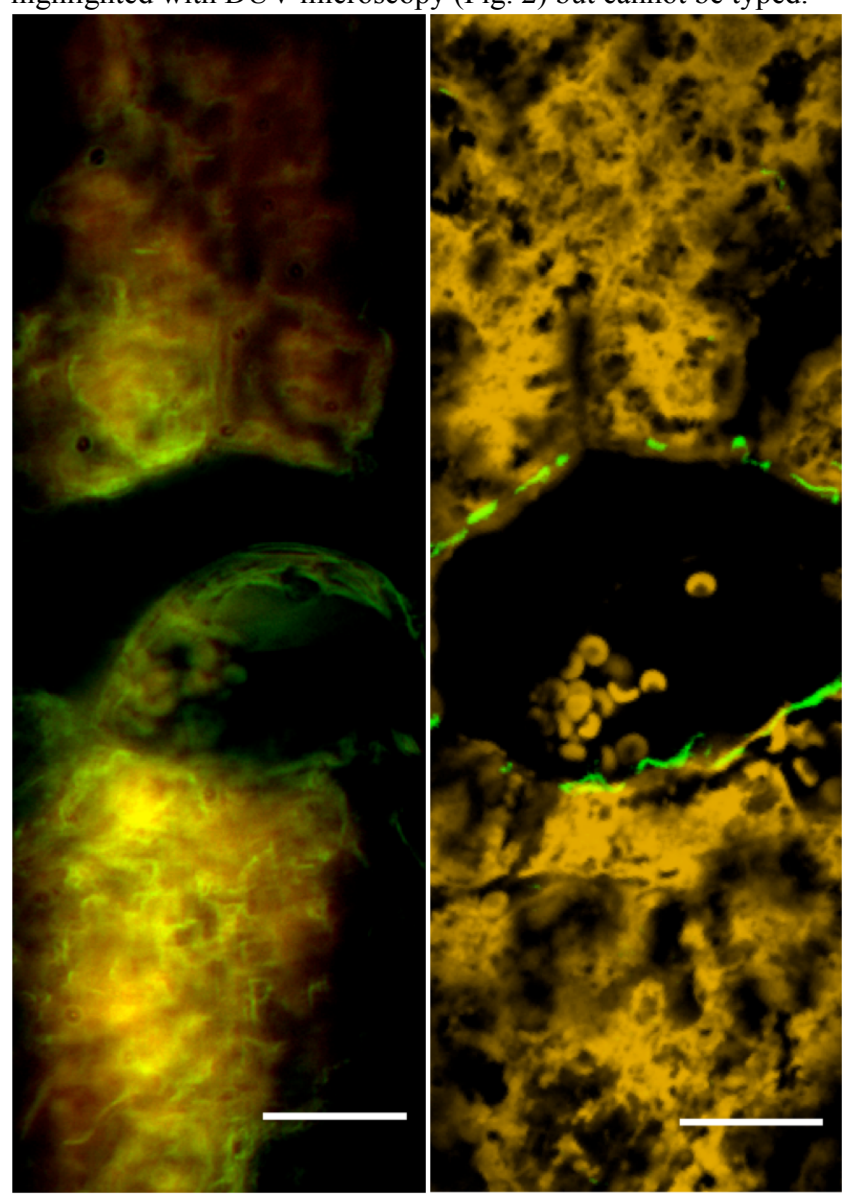

10 Figure 3. DUV fluorescence image (left), collagen is in green, amino acids in red and SHG image (green) merged with TPEF (yellow) (right) of healthy mice liver (excitation: $330 \mathrm{~nm}$; emission: $412-438 \mathrm{~nm}$; targeting regions of collagen localization). Scale bar $20 \mu \mathrm{m}$.

We observe collagen type I and II surrounding the central vein 15 but also cells in DUV while in SHG only around the vein (Fig. 3). Ultraviolet excited fluorescence (either two-photon or single photon, yellow in Fig. 3) permits also to visualize hematocytes that remained trapped inside the vein, only DUV microscopy shows the network of fibres surrounding those cells.

${ }_{20}$ DUV microscopy illustrates the distribution of endogenous compounds in the liver tissue. The filters have been chosen close to regions of fluorescence maxima of tryptophan, collagen and elastin. The band pass filter at $327-353 \mathrm{~nm}$ (excitation at 290 $\mathrm{nm}$ ) was used to visualize tryptophan occurrence in the biological 25 sample (Fig. 3). It reveals protein distribution in the liver with different pathologies. The differences in signal intensities between two channels (red filters at $327-353 \mathrm{~nm}$ and green at $412-438 \mathrm{~nm}$ ) allow the detection of collagen abundance.
Collagen type IV forms a structural basis of cell membranes. ${ }_{30}$ Because it is a globular protein, it can be imaged by DUV microscopy only, not by SHG. ${ }^{3}$ Therefore, DUV fluorescence imaging provides deeper knowledge concerning collagen repartition in tissues (Fig. 3).

Collagen fibres develop mostly in vascular regions. Collagen 35 fibres have spring-like structures that are randomly oriented surrounding cell membranes. But close to veins or arteries collagen fibres are co-directed around the tubular objects (Fig. 3).

\section{Conclusions}

Two-photon microscopy found a broad range of applications in 40 biology, for example, multi-photon excitation imaging of neuron activity, cancer research studies of angiogenesis, metastasis and embryo development visualization. ${ }^{10}$ On the other hand, Deep UV light (200-300 nm) and near UV light (300-400 nm) are used for excitation of many endogenous compounds, which are 45 responsible for native tissue fluorescence. The endogenous fluorophores present in most biological tissues are: NADH coenzymes which is present in mitochondria, chloroplasts, peroxisomes, cytosol and is involved in cellular metabolism, presents an excitation maxima at 260 and $345 \mathrm{~nm}^{9,17}$, aromatic 50 autofluorescent amino acids: tryptophan (excitation maxima 220 and $277 \mathrm{~nm})$, tyrosine $(274 \mathrm{~nm})$, phenylalanine $(257 \mathrm{~nm})$ compose many proteins. Furthermore, flavins, collagen, elastin, FAD and lipopigments are endogenous chromophores in tissues under UV light excitation. ${ }^{9,18}$

${ }_{55}$ Development of new methods for liver pathological tests is on front line of actuality in many known researches. SHG microscopy has high potential for characterization of fibrotic stages in liver and was approved the Fibrosis-Metavir test ${ }^{3}$. Moreover, it has been shown that collagen fibres detection in 60 liver by non-linear microscopy provides better sensitivity compared to conventional staining methods. ${ }^{19}$ Combination of SHG and synchrotron light excitation autofluorescence microscopies for biological tissue analysis has not been reported in any other study. Therefore, this research work has a significant ${ }_{65}$ value not only as a tool for the characterization of pathological disorders, but also demonstrates the compatibility of these techniques for analyzing the same sample. While SHG permits a fine localization and quantification of fibrillar collagen type I and II, DUV fluorescence microscopy images all collagen crosslinks 70 without discrimination. While this loss in selectivity could be considered as a disadvantage, it may also be seen as an advantage because comparison between DUV and SHG images permits to localize collagens of other types than I and II.

Due to the potential presented for liver tissues fibrillar 75 characterization, the mentioned microscopy methods could be applied to study pathological disorder caused by non-alcoholic fatty liver disease (NAFLD). Those disorders are expressed in an unordinary accumulation of lipid droplets in the liver of nonalcoholic patients. $^{20}$ NAFLD is classified between simple 80 steatosis (fatty liver) and non-alcoholic steatohepatitis (NASH). The histopathological spectrum of NAFLD extends from steatosis through NASH to advanced fibrosis, cirrhosis and hepatocellular carcinoma (HCC). While steatosis refers to lipids accumulation, the key factor concurs to favour NASH are oxidative stress ${ }_{85}$ culminating with liver injury and inflammation. ${ }^{21}$ It has been 
shown that the simple hepatic steatosis confers a long-term prognosis. It is a process reversible, considered innocuous in its pure form. Indeed patients without evidence of NASH rarely progressed to cirrhosis ${ }^{22,23}$, whereas the disease progression 5 occurs in $43 \%$ of patients with $\mathrm{NASH}$, and progression to cirrhosis may occur in $28 \%$ of these cases ${ }^{23,24}$. In addition, HCC has long been described to arise on a cirrhotic liver. Recent data however show that some patients with NASH can progress to HCC bypassing the stage of cirrhosis ${ }^{25}$. Therefore, NASH is now

10 emerging as a leading risk factor owing to the epidemic of obesity and type 2 diabetes mellitus becoming major public health problem worldwide. Despite the major public health concern of NAFLD, it is currently impossible by the usual histological methods to identify at early stage patients that will

15 progress from steatosis to NASH. This justifies the need of new methods to quantitatively assess the early biochemical changes related to this pathology.

At present, the gold standard for the diagnosis of liver pathology is liver biopsy. These liver probes are analyzed mainly by visual 20 inspection after appropriate staining protocols. Although rich on information, from the clinical point of view, histopathology of liver is prone to intra- and inter-observer variability, which can yield poor reproducibility even when performed by experts. In the study of El-Badry et al. ${ }^{26}, 4$ pathologists assessed the features of $25 \mathrm{NAFLD/NASH}$ and a strong disagreement was found for all parameters including the overall diagnosis. Evaluation of NAFLD is therefore strongly observer-dependent and seems weakly reproducible. The incapacity of objectively assess the NASH and identify at an early stage patients that will progress from steatosis 30 to NASH justifies the need of new methods to quantitatively assess the biochemical changes related to this pathology. Recently, joint application of time-of-flight Secondary Ion Mass Spectrometry, DUV Microspectroscopy and infrared microspectroscopy for lipid, protein, sugar and nucleic acid

35 mapping on liver samples has been developed. ${ }^{1,27}$ Early studies showing SHG microscopy as a tool for liver-pathology examination, were reported. ${ }^{28,29}$ Since then, several research groups assessed the method as a perspective technique bringing more sensitive results than conventional fibrosis scoring 40 methods $^{3,9}$ and proposed a quantification method. ${ }^{20,30}$ Because SHG microscopy presents perspectives as a fast, non-destructive, quantitative technique, which eliminates human factors in liver analysis procedure, its combination with DUV single photon fluorescence microscopy looks even more promising.

\section{${ }_{45}$ Notes and references}

${ }^{a}$ DISCO Beamline, Synchrotron SOLEIL, F-91192 Gif sur Yvette, France. Fax: 331 69359456; Tel: +331 69359655; E-mail: matthieu.refregiers@synchrotron-soleil.fr

${ }^{b}$ actual adress: EPFL / STI-IBI-STI / LBP - CH - 1015 Lausanne, 50 Switzerland

${ }^{c}$ Inserm U785, F-94800 Villejuif, France

${ }^{d}$ Univ. Paris-Sud 11, UMR-S785, F-94800 Villejuif, France

Acknowledgments

Vitalijs Zubkovs is supported by ERASMUS MUNDUS student grant

55 We acknowledge Synchrotron SOLEIL support through projects \#20100064, 201000181, 20100949 and 20110131.

1. G.A. Di Lullo, S.M. Sweeney, J. Körkkö, L. Ala-Kokko and J.D. San Antonio, Journal of Biological Chemistry, 2001, 277, 4223-4231.
2. P. Campagnola, M. Wei, A. Lewis and L.M. Loew, Biophysical Journal, 1999, 77, 3341-3349.

3. L. Gailhouste, Y. Le Grand, C. Odin, D. Guyader, B. Turlin, F. Ezan, Y. Désille, T. Guilbert, A. Bessard, C. Frémin, N. Theret, and G. Baffet, Journal of Hepatology, 2010, 52, 398-406.

4. P. Campagnola, A. Millard, M. Terasaki, P.E.. Hoppe, C.J. Malone and W.A. Mohler, Biophysical Journal, 2002, 81, 493-508.

5. J. A. Palero, H. S. de Bruijn, A. van der Ploeg van den Heuvel, H. J. C. M. Sterenborg, and H. C. Gerritsen, Biophysical Journal, 2007, 93, 992-1007.

6. G. Filippidis, E. J. Gualda, M. Mari, K. Troulinaki, C. Fotakis, and N. Tavernarakis, Micron, 2009, 40, 876-880.

7. X. Chen, O. Nadiarynkh, S. Plotnikov, and P. J. Campagnola, Nature Protocols, 2012, 7, 654-669.

8. F. Jamme, S. Villette, A. Giuliani, V. Rouam, F. Wien, B. Lagarde, and M. Refregiers, Microsc Microanal, 2010, 16, 507-514.

75 9. F. Jamme, S. Kascakova, S. Villette, F. Allouche, S. Pallu, V. Rouam, and M. Refregiers, Biology of the Cell, 2013, 105, 277-288.

10. W. R. Zipfel, R. M. Williams, and W. W. Webb, Nat Biotechnol, 2003, 21, 1369-1377.

11. A. Giuliani, F. Jamme, V. Rouam, F. Wien, J.-L. Giorgetta, B. Lagarde, O. Chubar, S. Bac, I. Yao, S. Rey, C. Herbeaux, J.-L. Marlats, D. Zerbib, F. Polack, and M. Refregiers, J Synchrotron Rad, 2009, 16, 835-841.

12. A. Edelstein, N. Amodaj, K. Hoover, R. Vale, and N. Stuurman, Current protocols in molecular biology, 2010, 92, 14.20.1-14.20.17.

85 13. A. A. de Aro, B. de Campos Vidal, and E. R. Pimentel, Micron, 2012, 43, 205-214.

14. P. P. Provenzano and R. Vanderby Jr., Matrix Biology, 2006, 25, 7184.

15. T. A. Theodossiou, C. Thrasivoulou, C. Ekwobi, and D. L. Becker, Biophysical Journal, 2006, 91, 4665-4677.

16. W. Zipfel, R. Williams, R. Christie, A. Nikitin, B. Hyman, and W. Webb, Proc. Nat. Acad. Sci. U.S.A., 2003, 100, 7075-7080.

17. M. R. Kasimova, J. Grigiene, K. Krab, P.H. Hagedorn, H. Flyvbjerg, ..E. Andersen and I.M. Moller, The Plant Cell, 2006, 18, 688-698.

95 18. A. G. Wagnières, W. M. Star, and B. C. Wilson, Photochem Photobiol, 1998, 68, 603-632.

19. D. C. S. Tai, N. Tan, S. Xu, C. H. Kang, S. M. Chia, C. L. Cheng, A. Wee, C. L. Wei, A. M. Raja, G. Xiao, S. Chang, J. C. Rajapakse, P. T. C. So, H.-H. Tang, C. S. Chen, and H. Yu, J. Biomed. Opt., 2009, 14, 044013.

20. P. Angulo, Hepatology, 2010, 51, 373-375.

21. C. P. Day and O. F. James, Gastroenterology, 1998, 114, 842-845.

22. M.R. Teli, O.F. James, A.D. Burt, M.K. Bennett and C.P. Day, Hepatology, 1995, 22(6), 1714-1719.

105 23. C.A. Matteoni, Z.M. Younossi, T. Gramlich, N. Boparai, Y.C. Liu and A.J. McCullough, Gastroenterology, 1999, 116(6), 1413-1419.

24. R.G. Lee, Hum. Pathol., 1989, 20 (6), 594-598.

25. K. Yasui, E. Hashimoto, Y. Komorizono, K. Koike, S. Arii, Y. Imai, T. Shima, Y. Kanbara, T. Saibara, T. Mori, S. Kawata, H. Uto, S. 110 Takami, Y. Sumida, T. Takamura, M. Kawanaka and T. Okanoue, Clin. Gastroenterol. Hepatol., 2011, 9(5), 428-433.

26. A.M. El-Badry, S. Breitenstein, W. Jochum, K. Washington, V. Paradis, L. Rubbia-Brandt, M.A. Puhan, K. Slankamenac, R. Graf and P.A. Clavien, Ann Surg, 2009, 250(5), 691-697.

115 27. V. W. Petit, M. Refregiers, C. Guettier, F. Jamme, K. Sebanayakam, A. Brunelle, O. Laprevote, P. Dumas, and F. Le Naour, Anal. Chem., 2010, 82, 3963-3968.

28. R. Bataller and D.A. Brenner, Journal of clinical investigation, 115, 209-218.

120 29. G. Cox, E. Kable, A. Jones, I. Fraser, F. Manconi, and M. D. Gorrell, Journal of Structural Biology, 2003, 141, 53-62.

30. T. Guilbert, C. Odin, Y. Le Grand, L. Gailhouste, B. Turlin, F. Ezan, Y. Désille, G. Baffet, and D. Guyader, Optics Express, 2010, 18, 25794-25807. 